\title{
Performance of RC Beams with or without FRP Strengthening Subjected to Impact Loading
}

\author{
Hong Hao and Thong Pham \\ Centre for Infrastructural Monitoring and Protection, School of Civil and Mechanical Engineering, Curtin University \\ Kent Street, Bentley, WA 6102, Australia \\ hong.hao@curtin.edu.au; thong.pham@curtin.edu.au
}

During its service life, some reinforced concrete (RC) structures might be subjected to impact loads such as rock fall, vehicle collision, ship impact, and accident dropping of heavy objects. Therefore impact resistance design is essential for the safety and serviceability of such structures. However, the impact behaviour of RC beams is not well understood yet, and methods for strengthening RC structures against impact loading are still very limited. In this study, experimental tests and numerical simulations were conducted to investigate the performance of RC beams with and without fibre reinforced polymer (FRP) strengthening subjected to impact loads. Fourteen rectangular RC beams were experimentally tested under a dropweight test apparatus and intensive numerical simulations are conducted by using LS-Dyna. The impact behaviour of RC beams is observed and compared with its counterpart under static loading. The unique impact response characteristics of RC beams are discussed. It was observed that under impact loadings the equilibrium of the beam was maintained primarily by the inertia resistance of the beam at early stage of the impact process, the contribution from the reaction forces is negligible. The shear failure was found to be critical under impact loads although the identical beam had the shear strength 4 times of the flexural strength and failed in the flexural mode under static loads. The plastic hinge induced by impact load significantly affects the impact behaviour of the RC beams regardless of the boundary conditions in relatively long beams. Strengthening RC beams with FRP can considerably reduce the impact responses in case of the both flexure- and shear-deficient beams. Therefore, strengthening RC beams with FRP is an effective method to improve their performance against impact loading.

Keywords: Impact behaviour, impact loading, fibre reinforced polymer (FRP), strengthening, RC beams. 